\title{
Sistema de monitoreo de consumo eléctrico con interfaz para teléfono inteligente
}

\author{
Alejandro Andrés Serapio Carmona ${ }^{1}$, Ismael Díaz Rangel², \\ Rodolfo Zola García Lozano ${ }^{1}$, Cuauhtémoc Hidalgo Cortés ${ }^{1}$ \\ ${ }^{1}$ Universidad Autónoma del Estado de México, Centro Universitario Ecatepec, México \\ ${ }^{2}$ Universidad Nacional Autónoma de México, Facultad de Estudios Superiores Aragón, México \\ andreserapio@hotmail.com, zolagarcialyahoo.com, \\ chidalgoc@uaemex.mx, ismael1099@hotmail.com
}

Resumen. El consumo eléctrico, es la cantidad de energía eléctrica que se ha utilizado a lo largo de un periodo definido, por la cual se debe pagar. El uso de la energía eléctrica está determinado por el estilo de vida de cada usuario, así como de la actividad para la que se destine su uso [1]. La empresa encargada de proveer la energía eléctrica cuenta con un sistema de cobro de tarifas que se incrementa a mayor consumo [2]. Esta información, se le da a conocer al usuario hasta que termina el periodo y se aplica el cobro. En este trabajo se describe el desarrollo de un sistema, cuya función principal es ser una herramienta que ayude a los usuarios a conocer fácilmente el estado del consumo eléctrico de manera anticipada a la fecha de corte; de esa manera, ellos podrán tomar las medidas pertinentes para utilizar racionalmente la energía eléctrica. Adicionalmente, el sistema brindará un estimado de la cantidad a pagar por el consumo realizado desde el inicio del corte hasta el momento actual. El módulo de monitoreo se comunica con un teléfono inteligente por medio de bluetooth, el control es realizado por un microcontrolador; para la medición de la corriente eléctrica, se utiliza el sensor ACS712 de efecto hall, el cual fue caracterizado para la medición de corriente alterna; además, se guarda un histórico de las mediciones para su análisis.

Palabras clave: consumo eléctrico, monitoreo, sensor de corriente.

\section{Power Consumption Monitoring System with Smartphone Interface}

\begin{abstract}
Electricity consumption is the amount of electrical energy that has been used over a defined period, for which it must be paid. The use of electrical energy is determined by the lifestyle of each user, as well as the activity for which its use is intended [1]. The company responsible for providing electricity has a system for charging fees that increases to higher consumption [2]. This information is made known to the user until the end of the period and the payment is applied. This work describes the development of a system, whose main function is to be a tool that helps users to easily know the state of electricity consumption in advance of the cutoff date; that way, they can take the appropriate measures to rationally use electric power. Additionally, the system will provide an estimate of the amount to be paid for the consumption made from the
\end{abstract}


beginning of the cut until the current time. The monitoring module communicates with a smartphone via bluetooth, the control is carried out by a microcontroller; for the measurement of electric current, the hall effect ACS712 sensor is used, which was characterized for the measurement of alternating current; In addition, a history of the measurements is kept for analysis.

Keywords: electricity consumption, monitoring, current sensor.

\section{Introducción}

La energía eléctrica es un recurso que ha brindado apoyo para el desarrollo de las sociedades. Cuando el uso de la energía eléctrica comenzó a brindar mayores comodidades a las personas, su uso se fue incrementando; tanto, que en la actualidad la energía eléctrica se encuentra en casi todas las actividades diarias de las personas: en el transporte, en la preparación de la comida, en la iluminación, comunicaciones, entretenimiento, trabajo, etc. Su uso es tan intenso, que se ha tenido la necesidad de buscar e invertir en tecnologías que produzcan energía eléctrica a partir del uso de recursos renovables, con la finalidad de poder abastecer la creciente demanda de energía eléctrica, pues al crecer, lo hacen también las actividades relacionadas a su producción; aun así, es necesario cuidar de los recursos energéticos que se utilizan para la producción de energía, por lo que los usuarios deberían utilizar eficientemente la energía eléctrica proporcionada [3]. Para que el usuario tenga información y con ello tomar medidas de gestión del uso de energía, este trabajo propone el desarrollo de una herramienta para informar a los usuarios en cualquier momento, el estado del consumo energético. Adicionalmente, el sistema proporciona al usuario información acerca de la tarifa en que se encuentra, el precio a pagar por dicha tarifa, y el tiempo restante para la fecha de corte. La interfaz está desarrollada en una aplicación móvil, que se enlaza fácilmente con el módulo de monitoreo (o adquisición) mediante bluetooth.

\section{Estado del arte}

Existen trabajos, que han propuesto una solución para conocer el estado del consumo eléctrico a través del uso de medidores digitales. En [4] se describe un prototipo de medidor electrónico que considera las pérdidas de energía eléctrica ocasionadas por robo de energía a través del uso de sensores de corriente, colocados en segmentos específicos del tendido eléctrico; adicionalmente, el sistema puede establecer una comunicación a través de internet con la empresa que suministra el servicio, donde envía información acerca del estado del consumo; además, se puede activar o desactivar el suministro de energía de manera remota. El sistema utiliza un microcontrolador como sistema de procesamiento y de control, una pantalla LCD para mostrar la información al usuario y un Ethernet Shield para establecer una comunicación a través de internet. $\mathrm{El}$ autor refiere que las pruebas realizadas mostraron valores que se pueden considerar aceptables. Una desventaja de este prototipo, es el tendido que se debe realizar para enviar la información por internet a través de una red alámbrica; además, en caso de que el usuario no cuente con una conexión a internet, él tendrá que encargarse de cubrir este servicio. Otro aspecto a resaltar, es que el usuario para poder conocer el estado del 
consumo, debe de ir hasta donde se encuentra el sistema para visualizar la información en la pantalla.

Un sistema que posee como objetivo brindar información acerca del estado del consumo antes de que llegue la fecha de corte, se encuentra descrito en [5], el trabajo describe el desarrollo de un sistema de monitoreo basado en un microcontrolador como dispositivo de procesamiento, un medidor de energía utilizando una bobina, un ESP8622 como medio de comunicación inalámbrica WiFi, y una interfaz web para visualizar la información obtenida. Una desventaja que presenta el sistema es la necesidad de contar con una conexión a internet todo el tiempo; por lo que, si el usuario no cuenta con servicio de internet, no podrá visualizar la información del consumo en la página web.

Por otro lado, el sistema propuesto cuenta con una interfaz de teléfono inteligente que se comunica con el módulo de monitoreo a través de bluetooth, por lo que no se necesita contar con internet; de esta manera, el usuario podrá obtener mediante una app información del consumo, estimado del monto a pagar y días restantes para la fecha de corte. Adicionalmente, la interfaz de la app permite configurar fecha de corte y montos de tarifas. Las mediciones son almacenadas en una memoria MicroSD a razón de 5 segundos para que el usuario pueda revisar el histórico de su consumo.

\section{Tarifas}

En México la Comisión Federal de Electricidad (CFE) es quien suministra la energía eléctrica, y el esquema de cobro está definido la Comisión Reguladora de Energía (CRE), quien determina la metodología del cálculo de las tarifas para el suministro básico (Artículo 139 de la Ley de la Industria Eléctrica) [1]. Para la Ciudad de México y zona metropolitana el esquema de cobro se muestra en la tabla 1.

Tabla 1. Tarifas aplicadas a CDMX y zona metropolitana.

\begin{tabular}{lcc}
\hline \multicolumn{1}{c}{ Consumo } & Precio x KWh $(\mathbf{M X N})$ & Rango[KWh] \\
\hline Consumo básico & $\$ 0.81$ & $0<75$ \\
Consumo intermedio & $\$ 0.98$ & $75<140$ \\
Consumo excedente & $\$ 2.87$ & $140<250$ \\
Alto Consumo Doméstico & $\$ 107$ (fijo) $+\$ 4.79$ & $>250$ \\
\hline
\end{tabular}

Para dar un contexto, si se tienen dos cargas de $100 \mathrm{~W}$ (como un par de luminarias) durante las 24 horas durante un mes, el consumo total es de 144 KWh $(200 \mathrm{~W}$ x $24 \mathrm{~h}$ x 30 días), por lo cual se deberá pagar (según la tabla 1) lo siguiente: (75 KWh x \$0.81) $+(65 \mathrm{KWh} \times \$ 0.98)+(4 \mathrm{KWh} \times \mathbf{2} .87)=\$ \mathbf{1 3 5 . 0 0}$

Si se rebasa el rango de $250 \mathrm{KWh}$, se aplica el cobro de tarifa doméstica de alto consumo (DAC); tomando el ejemplo anterior, pero con cuatro cargas de $100 \mathrm{~W}$, el consumo mensual sería de $288 \mathrm{KWh}$ por lo que se debe pagar: $\$ 107+(288 \mathrm{KWh} \mathrm{x}$ $\$ 4.79)=\$ 1486.50$, lo que equivale a 10 veces más el monto con respecto al caso anterior. De ahí la importancia de tener la información referente al consumo de manera fácil y puntual, y con ello elegir las medidas pertinentes para gestionar el consumo. 


\section{Metodología}

El sistema propuesto está conformado por varias etapas que se pueden apreciar en el diagrama de bloques de la figura 1. En la figura 2 se presenta el diagrama esquemático del sistema.

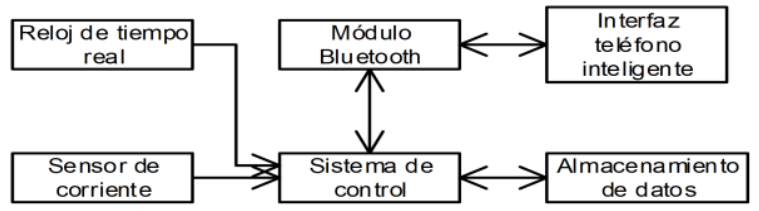

Fig. 1. Diagrama de bloques.

Sistema de control: Es el microcontrolador que se encarga de realizar todas las funciones lógicas del sistema, se utiliza un microcontrolador Atmega328 [6].

Sensor de corriente: Se utilizó el sensor de corriente de efecto Hall ASC712 versión para $30 \mathrm{~A}$, este bloque obtiene la información de la corriente utilizada, la cual se almacena en una variable [7].

Almacenamiento de datos: La información de fecha, hora y consumo, es almacenada en una memoria MicroSD, utilizando un MicroSD shield. Extrayendo la memoria, el usuario podrá revisar el histórico de su consumo, abriendo el archivo generado mediante un programa de hoja de cálculo.

Módulo Bluetooth: Realiza la comunicación con un teléfono inteligente para enviar y recibir datos, utilizando un módulo HC05 [8].

Interfaz para teléfono inteligente: Es una aplicación desarrollada para dispositivos Android mediante appInventor2 [9], la cual es la interfaz visual que permite comunicar al usuario y al sistema.

Reloj de Tiempo Real: De este componente se obtiene la información de la fecha, sirve para mantener un registro por fecha y hora de los valores obtenidos, utilizando un módulo RTC DS1307 [10].

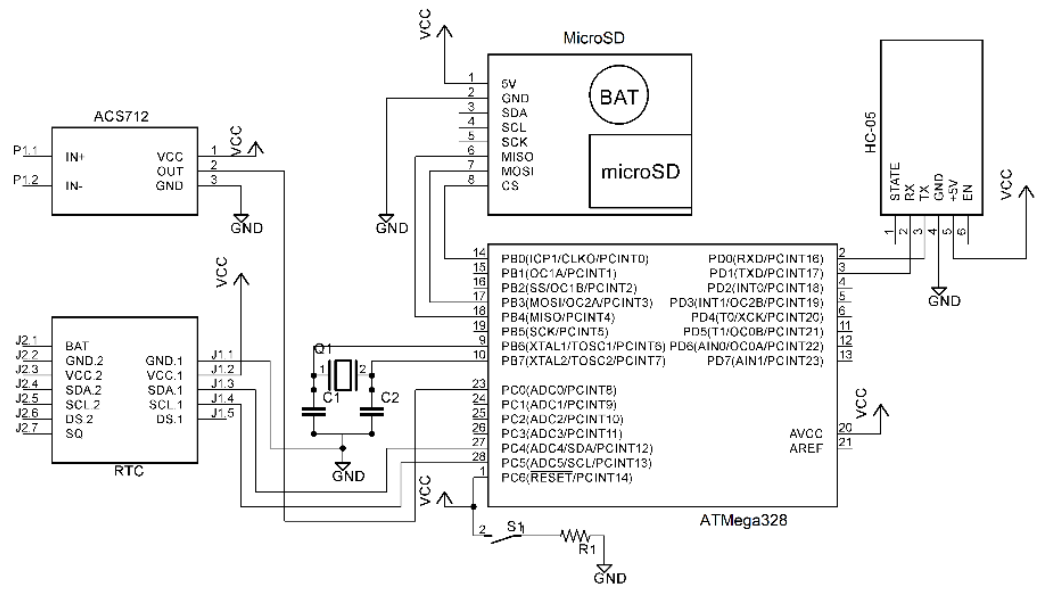

Fig. 2. Diagrama esquemático. 


\subsection{Sensor de corriente ACS712}

Es un sensor de corriente de efecto Hall, es de tipo invasivo, su funcionamiento consta de cuantificar la corriente a través de sus terminales (IN+, IN-), y utiliza el efecto Hall para producir en la salida un voltaje proporcional a la corriente que se encuentra circulando.

El valor en voltaje de la salida se observa en la figura 3, y muestra el comportamiento lineal del sensor.

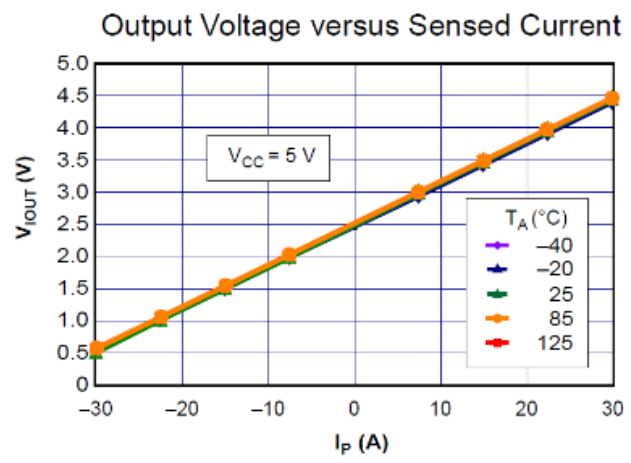

Fig. 3. Gráfica de voltaje vs corriente del ACS712.

\subsection{Medición de la corriente eléctrica}

Para la obtención del consumo, se realizaron experimentos para caracterizar el comportamiento de la salida del sensor en AC (Corriente Alterna). Para la caracterización, se analizó la salida del sensor con la ayuda de un osciloscopio. Aplicando una carga de $2300 \mathrm{~W}$ se obtuvo la señal mostrada en la figura 4, donde se puede apreciar que entrega un voltaje en directa, pero presenta variaciones que van desde un voltaje mínimo $880 \mathrm{mV}$ a un voltaje máximo $4.08 \mathrm{~V}$.

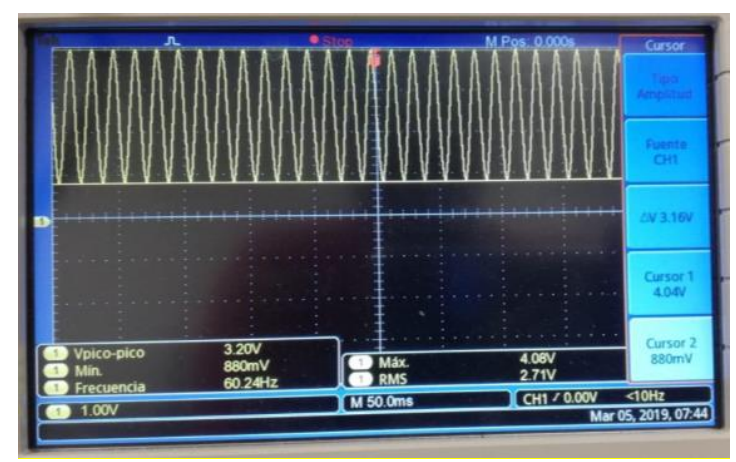

Fig. 4. Señal de salida del sensor con carga 2300W alimentada con AC.

Para calcular el valor de la corriente, se realizó un experimento en el que se conectaron diferentes cargas resistivas, y conociendo la linealidad del sensor, se empleó la ecuación de la recta para aplicar un análisis quedando definida como: 


$$
V=m I+B
$$

donde:

$\mathrm{V}=$ Voltaje de salida del sensor.

$\mathrm{I}=$ Corriente que circula por el sensor.

$\mathrm{B}=$ Voltaje cuando la corriente es cero.

$\mathrm{m}=$ pendiente $\mathrm{o}$ razón de cambio, estando definido por:

$$
m=\frac{V_{2}-V_{1}}{I_{2}-I_{1}} .
$$

De los valores mostrados por el fabricante y verificados experimentalmente, se extrajeron valores que se sustituyen en 2 y se obtiene:

$$
m=\frac{4.5-2.5}{30-0}=\frac{2}{30}=\frac{1}{15} \text {. }
$$

Ahora sustituyendo el valor de $m$ en (1) y utilizando la ordenada al origen, encontramos la expresión:

$$
V=\frac{1}{15} I+2.5
$$

Para obtener la potencia consumida es necesario trabajar con valores eficaces (RMS). Para obtener el voltaje RMS que entrega el sensor, se necesita obtener el voltaje de pico (Vp) máximo de un ciclo de la senoidal, por lo que se implementó un algoritmo que toma lecturas consecutivas durante 17 milisegundos con el convertidor analógico digital (dado que es la duración de un ciclo completo de una señal de $60 \mathrm{~Hz}$ ), y cada lectura realizada es comparada con la anterior, con el fin de obtener el valor mayor, el cual representa al Vp. Para el cálculo de la corriente RMS por definición se utiliza:

$$
I_{R M S}=\frac{I_{P}}{\sqrt{2}} \quad \text { despejando } I_{P}=I_{R M S} * \sqrt{2} .
$$

Sustituyendo (5) en (4) e indicado en lugar V al Vp, tenemos:

$$
V_{P}=\frac{1}{15}\left(I_{R M S} * \sqrt{2}\right)+2.5 \text { despejando } I_{R M S}=\frac{15\left(V_{P}-2.5\right)}{\sqrt{2}} .
$$

Para calcular la energía consumida de manera instantánea (que en realidad será la potencia consumida en un ciclo de la senoidal) se utiliza:

$$
P_{\text {ins }}=V_{R M S} \times I_{R M S}
$$

donde:

$P_{\text {ins }}=$ Potencia instantánea

$\mathrm{V}_{\mathrm{RMS}}=$ Voltaje eficaz de la fuente de alimentación.

$\mathrm{I}_{\mathrm{RMS}}=$ Corriente eficaz obtenida en (6).

Para conocer el acumulativo de energía consumida se calcula con:

$$
P_{\text {consumida }}=\frac{P_{\text {ins }}}{\Delta t},
$$

donde $\Delta \mathrm{t}$ es igual a 3600 , se debe a que se definió que cada lectura fuera tomada en intervalos de un segundo. Por la tanto, corresponde a 1/3600 parte de lo consumido en 1 hora. 
El algoritmo programado para la carga de valores iniciales (tarifas y fecha de corte) almacenados en la eeprom del microcontrolador, estimación de la potencia instantánea, la transmisión de datos hacia la aplicación se observa en el diagrama de la figura 5.

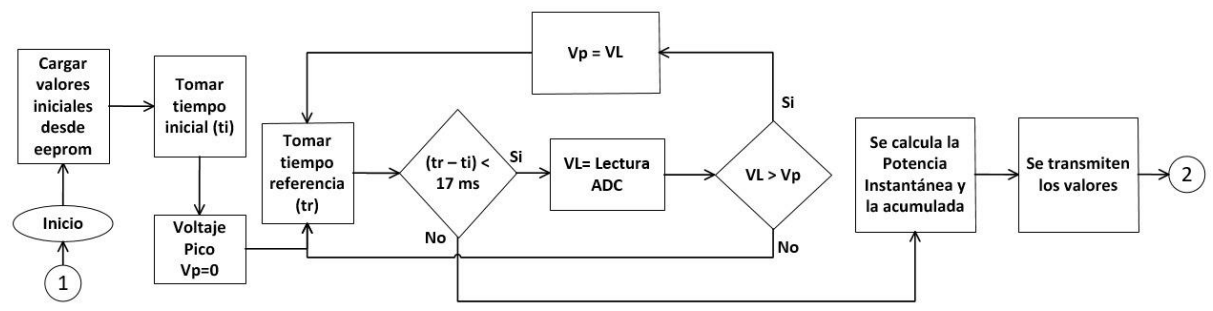

Fig. 5. Diagrama de flujo para obtener la potencia instantánea y su transmisión.

Para poder realizar las lecturas cada segundo, se desarrolló un algoritmo (figura 6) que asegura que haya transcurrido un segundo entre cada lectura, así se toman 3600 lecturas y sumatorias en el intervalo de una hora. Además, en esta sección, se monitorea la recepción de datos provenientes de la aplicación para la reconfiguración de parámetros y su almacenamiento en la eeprom.

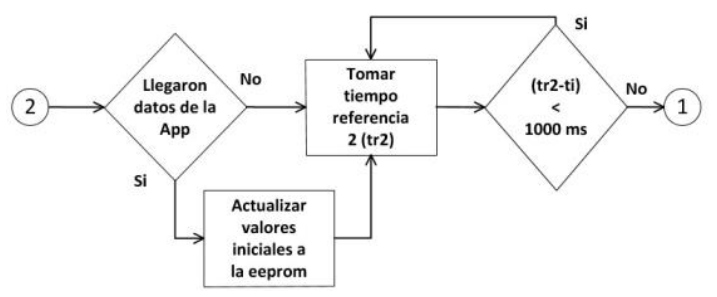

Fig. 6. Diagrama de flujo para tomar lectura cada segundo y reconfiguración de parámetros.

\subsection{Aplicación móvil}

La aplicación tiene como objetivo servir de interfaz visual y de configuración entre el usuario y el sistema, el funcionamiento de la aplicación se muestra en el diagrama de flujo de la figura 7.

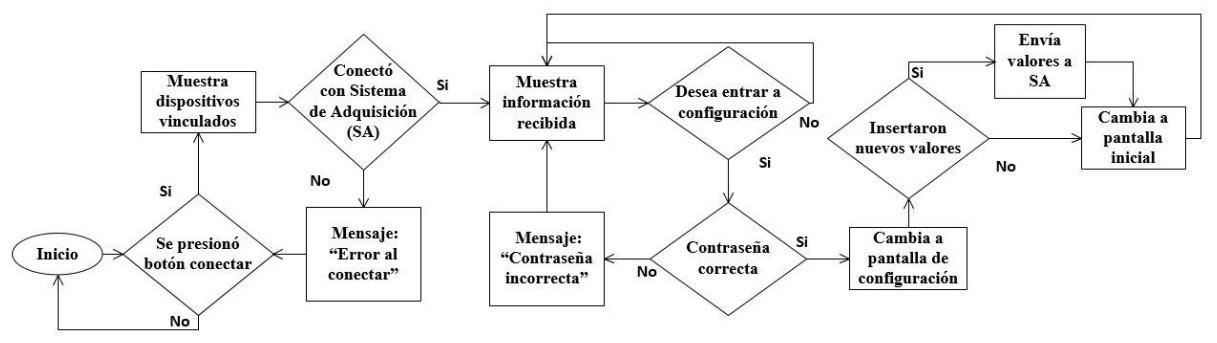

Fig. 7. Diagrama de flujo de la interfaz de la aplicación móvil.

Al ejecutar la aplicación (ver figura 8), la interfaz muestra al usuario la información del consumo, cuánto se va a pagar y cuántos días hacen falta para la fecha de corte. 
Para visualizar la información, es necesario pulsar el botón conectar, con el que se enlaza la aplicación con el módulo de monitoreo a través de una comunicación por bluetooth. Cuando se presiona el botón de configuración se solicitará el ingreso de un código de seguridad, con lo que se accederá a la pantalla de la figura 9, los campos corresponden a las tarifas vigentes y la fecha de corte; el botón enviar actualiza la información del sistema, lo cual es importante para establecer la información que se presentará en la pantalla inicial referente al consumo.

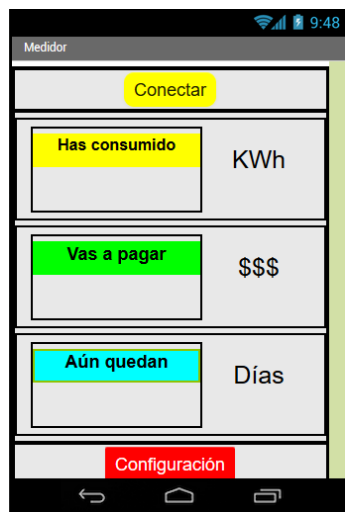

Fig. 8. Pantalla inicial.

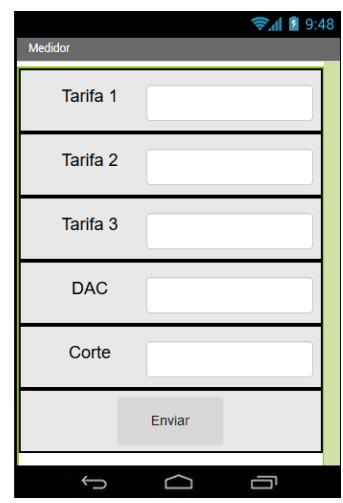

Fig. 9. Pantalla de configuraciones.

\section{Pruebas y resultados}

A continuación, se muestran los resultados de medir corrientes eficaces sobre diferentes cargas (tabla 2), todas ellas utilizando un voltaje de alimentación nominal de 127 VRMS, utilizando el sistema propuesto (SP) y un multímetro calibrado:

Tabla 2. Resultados de mediciones de corrientes.

\begin{tabular}{cccc}
\hline Carga & Amperímetro [A] & SP [A] & Diferencia [\%] \\
\hline Lámpara 40 watts & 0.336 & 0.38 & 14 \\
Lámpara 100 watts & 0.58 & 0.6 & 4.5 \\
Plancha 1100W & 8.65 & 8.48 & -1.9 \\
Estación de soldar & 5.82 & 5.46 & -6.5 \\
Plancha 2300W & 17.21 & 16.99 & -1.25 \\
Parrilla eléctrica & 8.65 & 8.36 & -3.25 \\
& & Promedio*: & -1.68 \\
\hline
\end{tabular}

* Diferencia promedio sin considerar cargas pequeñas

Se pudo comprobar que para cargas superiores a 0.3 Amper los resultados son bastante aproximados, no tanto así para cargas inferiores; al ser un sistema para su implementación en hogares, no se considera una situación relevante, ya que los consumo suelen ser muy superiores a $0.3 \mathrm{~A}$. Además, se debe considerar que existen fluctuaciones en la red de energía de la compañía proveedora y que eso también es 
causa de las diferencias, aunque se trató de realizar las lecturas de manera simultánea, ese factor de error es a considerarse.

Para las pruebas de consumo instantáneo, se conectó el sistema propuesto en el centro de cargas de un hogar en serie con un sistema comercial [SC] de medición de consumo eléctrico con la capacidad de recolección de mediciones. Las mediciones se realizaron durante 7 días, obteniéndose casi tres mil muestras, la figura 10 muestra los resultados comparativos de los sistemas. Los resultados entregaron un valor promedio de mediciones de potencia instantánea para el sistema propuesto de $3.72 \mathrm{~W}$, y para el sistema comercial de $3.7235 \mathrm{~W}$, lo que implica una diferencia aproximada del $0.1 \%$.

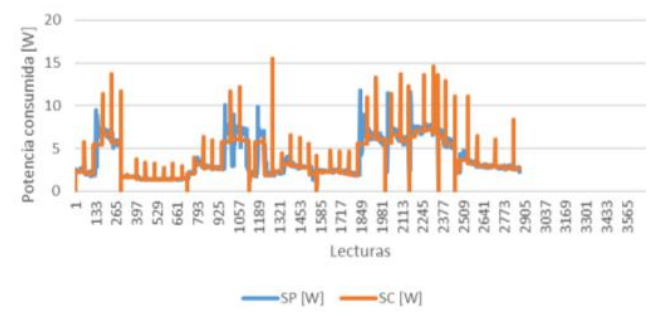

Fig. 10. Comparativa de mediciones.

Para determinar la equivalencia estadística, se aplicó a los datos el método de concordancia propuesto por Bland-Altman [11], el cual muestra una gráfica (figura 11) que indica niveles de correlación entre dos métodos de medición que observan un mismo fenómeno.

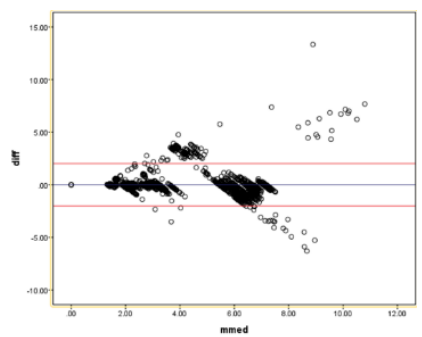

Fig. 11. Gráfica de Bland-Altman de las mediciones entre los dos sistemas.

Se puede observar como la gran mayoría de los datos se encuentran entre las líneas rojas (intervalo de confianza), lo cual permite determinar que los sistemas estadísticamente son equivalentes en sus mediciones.

Tabla 3. Resultados prueba medición consumo.

\begin{tabular}{ccc}
\hline Fecha & CFE [KWh] & SP [KWh] \\
\hline $17 / 06 / 2019$ & 1 & 1 \\
$18 / 06 / 2019$ & 0 & 0 \\
$19 / 06 / 2019$ & 1 & 1 \\
$20 / 06 / 2019$ & 1 & 1 \\
$21 / 06 / 2019$ & 0 & 0 \\
$24 / 06 / 2019$ & 1 & 1 \\
$25 / 06 / 2019$ & 1 & 1 \\
\hline
\end{tabular}


Para el consumo acumulado, se conectó el sistema a la salida del centro de cargas de un hogar, y se realizaron lecturas de manera manual durante 7 días con respecto al medidor de la CFE (mismo horario entre cada día), se calculó el consumo registrado en términos de KWh, ya que es la unidad de medida del medidor, y se compararon con el sistema propuesto, la tabla 3 muestra los resultados.

Se puede observar que los resultados son idénticos, por lo que se comprueba la eficacia del sistema para medir el consumo eléctrico en KWh.

\section{Conclusiones}

Se desarrolló el sistema de medición con base a un microcontrolador Atmega328, donde se logró la implementación de algoritmos para la adquisición y procesamiento de datos, así como del control de almacenamiento y transmisión de datos. Cumpliendo de manera precisa con las cotas de tiempos necesarias para la adecuada cuantificación de consumo eléctrico.

Además, se diseñó una aplicación para el sistema operativo Android, la cual funciona como interfaz visual y de configuración, para que el usuario pueda observar la información del consumo proporcionada por el sistema, y también permite configurar algunas variables como tarifas y fecha de corte.

Se llevó a cabo el estudio del funcionamiento en AC del sensor de corriente; esto, a partir del análisis de cargas resistivas conocidas alimentadas con AC, se modelo un algoritmo para la interpretación de las lecturas cuando se trabaja con AC, con esa información, se calculó la potencia instantánea y a partir de esta última, se obtuvo el valor del consumo eléctrico, expresado en KWh.

Se realizó un análisis estadístico de la fiabilidad del sistema con respecto a un sistema comercial, obteniendo valores dentro del intervalo de confianza que indican la equivalencia de mediciones entre ambos sistemas, lo que indica que el sistema posee un grado de aproximación similar a un medidor de tipo comercial.

\section{Referencias}

1. Comisión Federal de Electricidad (CFE): https://app.cfe.mx/Aplicaciones/CCFE/Tarifas/ Tarifas/Tarifas_casa.asp?Tarifa=DACTAR $1 \&$ anio=2018. (Último acceso: 22 enero 2019)

2. Escoto Castillo, A., Sánchez Peña, L., Pérez Guardián, G.: Hogares y energía eléctrica en México. Revista Espinhaço, vol. V, pp. 30-43 (2017)

3. Riera, P.: Manual de economía ambiental y de los recursos naturales. Madrid, Parainfo (2005)

4. Gaibor, E.R. et al: Implementación de un prototipo de medidor de energía eléctrica residencial considerando la reducción de pérdidas no técnicas por hurto. Publicando, pp. 66-82 (2018)

5. Díaz Pérez, P., Clemente Arce, V.G., Ortiz Muñiz, J.A.: Sistema embebido basado en el monitoreo de consumo de energía eléctrica de electrodomésticos para el control del gasto. Tecnología Digital 8(1), 69-80 (2018)

6. Microchip: ATmega328 Datasheet. http://ww1.microchip.com/downloads/en/ DeviceDoc/Atmel-7810-Automotive-Microcontrollers-ATmega328P_Datasheet.pdf (Último acceso: 14 noviembre 2018) 
7. Allegro MicroSystems, Inc., «Allegro MicroSystems,» https://www.google.com.mx/url?sa=t\&rct=j\&q=\&esrc=s\&source=web\&cd=2\&ved=2ah UKEwig2OHr9Y_jAhXDXc0KHXINACEQFjABegQIABAC\&url=https\%3A\%2F\%2F www.allegromicro.com\%2F \%2Fmedia\%2Ffiles\%2Fdatasheets\%2Facs712datasheet.ashx\&usg=AOvVaw1LkWwxiA9ZbTsL_UhbCLAp. (Último acceso: 5 octubre 2018)

8. Makezine: Make Magazine. 1 marzo 2014. https://cdn.makezine.com/uploads/ 2014/03/hc_hc-05-user-instructions-bluetooth.pdf. (Último acceso: 18 febrero 2019)

9. MIT: MIT APP INVENTOR. https://appinventor.mit.edu/explore/ai2/tutorials.html. (Último acceso: 24 enero 2019)

10. Maxim integrated: https://datasheets.maximintegrated.com/en/ds/DS1307.pdf. (Último acceso: 14 noviembre 2018)

11. Altman, D., Bland, J., Altman, D.G., Bland, J.M.: Measurement in medicine: the analysis of method comparison studies. Journal of the Royal Statistical Society: Series D (The Statistician), vol. 32, no 3, pp. 307-317 (1983) 